\title{
Qui dit Noël dit cadeaux : modèle, variation et idées conventionnelles
}

\author{
Kentaro Koga* \\ Tokyo University of Foreign Studies, Tokyo, Japon
}

\begin{abstract}
Résumé. Cette recherche vise à donner une description sur l'usage de la construction du type qui dit $X$ dit $Y$ en français d'aujourd'hui, en analysant des données observées dans des corpus et sur internet. Dans la première partie, nous nous intéresserons à la structure interne de la construction. Dans cette séquence, nous pouvons constater une certaine liberté de modification, alors que certains constituants semblent figés. Afin d'expliquer cette cohabitation entre la partie figée et la partie libre, il est nécessaire de supposer un modèle de composition dont certaines cases sont préalablement spécifiées. Ensuite, afin de connaître la nature de la combinaison lexicale pour les cases $\mathrm{X}$ et $\mathrm{Y}$, nous analyserons les données attestées sur internet. En ce qui concerne la structure interne observée, il est intéressant de noter les trois points suivants : 1) le pronom relatif qui sans antécédent est très rare en français contemporain, sauf dans la construction qui dit $X$ dit $Y$; 2) les insertions d'adverbes sont possibles, notamment ceux de certitude ou d'ajout et 3) la combinaison lexicale XY dépend largement des idées considérées comme conventionnelles, ce qui reflète l'aspect socio-culturel de la langue.
\end{abstract}

\begin{abstract}
Qui dit Noël dit cadeaux : model, variation and conventionalized ideas. This research aims to describe the usage of qui dit $X$ dit $Y$ type construction in today's French. In the first half of the article, we argue that the formation of this type of construction is realized by a model of composition which enables to coexist the archaic frozen usage and the underspecified sections. In the second half of the article, we analyze the examples that we collected from French corpora and from the Internet. The results of our analysis point out that : 1) the usage of the relative pronoun qui without antecedent is rare in contemporary French ; 2) it is possible to insert an adverb (adverbs of certainty or adverbs of addition, in particular), and 3) the lexical combination of $\mathrm{X}$ and $\mathrm{Y}$ depends on the idea that is considered as conventionalized, which reflect the sociocultural aspect of the language.
\end{abstract}

\section{Introduction}

La séquence du type qui dit $X$ dit $Y$ (e.g. qui dit Noël dit cadeaux) permet aux locuteurs d'exprimer un enchaînement conceptuel fort des deux éléments (i.e. Noël et cadeaux). Il ne

\footnotetext{
*kentkoga@gmail.com
} 
s'agit pas d'un syntagme libre, car sa structure interne ne correspond pas complètement aux règles grammaticales de la langue d'aujourd'hui. Cela ne signifie pour autant pas un « figement absolu » (cf. Mejri 2005 : 186), car nous pouvons constater une certaine liberté de modification, notamment pour le choix lexical sur les cases $\mathrm{X}$ et Y. Étant donné que les positions modifiables semblent déterminées par avance pour ce type de séquence, la liberté de modification est assurée non pas d'une manière temporaire ou ad hoc, mais plutôt de façon stable. Nous appelons cette séquence « construction ». Une construction est basée sur un modèle (ou schéma) de composition qui organise le cadre de formation.

L'objectif de cette recherche est non seulement d'expliquer comment le modèle de composition organise la structure interne de cette construction, mais aussi d'analyser la variation de la construction, notamment la possibilité de modification syntaxique et le choix lexical. En effet, la construction en question (désormais qui dit $X$ dit $Y$ ) accepte certaines modifications syntaxiques. Ainsi, il est possible de dire qui dit Noël ne dit pas forcément cadeaux, mais il y a des limites à la modification syntaxique. En ce qui concerne le choix lexical, la possibilité semble relativement large. Mais s'il existe d'autres éléments que cadeaux pour Y, de quoi s'agit-il concrètement ? Nous examinerons ces questions en analysant les données collectées sur internet.

\section{Modèle de composition : structure interne}

\subsection{Caractère proverbial}

Nous pouvons constater dans la construction qui dit $X$ dit $Y$ un caractère proverbial, mais en général, les séquences adoptant cette formule ne sont pas considérées comme un "vrai » proverbe. Nous pourrions les qualifier d'énoncés pseudo-proverbiaux.

Le caractère proverbial de la construction provient de sa structure interne. Mejri (2001) propose la notion de la «matrice idéelle» (p.11) comme un mécanisme garantissant la cohérence sémantique des énoncés proverbiaux. À la base de cette idée, il existe un «binarisme structurel»(p.10), notamment la structure corrélative, que représentent les énoncés proverbiaux. Ainsi, en juxtaposant les deux syntagmes opérés par tel, les énoncés du type tel $X$, tel $Y$ acquièrent leur statut proverbial :

(1) a. Tel père, tel fils

b. Tel maître, tel valet

c. Tel auteur, tel livre

Les énoncés qualifiés de pseudo-proverbiaux se forment de la même façon. Par exemple, l'énoncé tel Président, tel Premier ministre n'est pas enregistré dans les dictionnaires en tant que proverbe, certes, mais nous pouvons tout de même le considérer au moins comme quelque chose de proverbial. C'est grâce à l'application du même modèle (i.e. tel $X$, tel $Y$ ) que cet énoncé prend son caractère proverbial.

Par ailleurs, il est aussi possible de supposer un binarisme ou une compatibilité sémantique pour les éléments remplissant les cases X et Y. Cependant, cela ne semble pas être une condition nécessaire pour la réalisation du caractère proverbial. En effet, il existe des combinaisons de deux éléments moins attendues (e.g. tel père, tel chat), mais cela n'empêche pas cet énoncé d'être (pseudo-)proverbial ${ }^{1}$.

À cet égard, il n'y a pas, stricto sensu, de différence entre les « vrais » proverbes et les énoncés pseudo-proverbiaux. La seule différence serait l' "authenticité » (autrement dit, une question d'institutionnalisation) des proverbes. Cela revient quasiment au même problème que la présence ou non d'un terme dans les dictionnaires, et peut être mesuré 
jusqu'à un certain degré en faisant précéder l'expression comme dit le proverbe (cf. Schapira $2000: 89-90$, Tamba $2000: 113-115)$ :

(2) a. Comme dit le proverbe, tel père, tel fils.

b. ?Comme dit le proverbe, tel Président, tel Premier ministre.

c. ?Comme dit le proverbe, tel père, tel chat.

Hormis les utilisations volontairement humoristiques de l'énonciation, moins l'énoncé est attesté comme proverbe, moins la tournure comme dit le proverbe s'y attache facilement.

Au niveau référentiel, il est intéressant de noter que les deux syntagmes nominaux (i.e. $\mathrm{X}$ et Y) ne réfèrent pas concrètement à un objet (Schapira $2000: 85$, Anscombre 2003 : 161-

162). Dans ce type d'énoncé, les constituants nominaux sont souvent privés d'article, ce qui signifie que les référents ne sont pas complètement actualisés. Cette absence d'article est maintenue dans les énoncés pseudo-proverbiaux.

Or, contrairement à tel $X$, tel $Y$, il est difficile d'identifier la trace de l'analogie pour qui dit $X$ dit $Y$. Certes il existe de nombreux proverbes portant la structure qui V1 V2 (e.g. qui dort dîne, qui s'y frotte s'y pique etc.), ainsi que leurs versions « détournées » (e.g. qui dîne tard dort $\mathrm{mal}^{2}$ ), mais autant que nous sachions, il n'y a pas de proverbe considéré comme «authentique » portant la structure qui dit $X$ dit $Y$. Alors, d'où viennent les séquences comme qui dit Noël dit cadeaux? Notre hypothèse porte sur la possibilité de développer un nouveau modèle à partir d'un modèle de composition existant, plutôt qu'une forme particulière. Autrement dit, une fois établi un modèle de composition (dans ce cas, qui VI $V 2$ ), il est possible de former de nouvelles séquences indépendamment des formes existantes. À ce stade, il ne s'agit plus d'une simple analogie d'une forme à une autre, mais d'une formation basée sur un système plus abstrait.

\subsection{Qui dit X dit $Y$ : cohabitation des éléments figés et des parties libres}

\subsubsection{Pronom relatif qui sans antécédent}

Afin d'examiner la structure concrète du modèle, il est incontournable de revenir sur l'usage archaïque de qui. En français contemporain, hormis quelques types de textes particuliers (e.g. les définitions données dans les dictionnaires : «qui participe à quelque chose » pour le mot participant), l'antécédent est obligatoire pour ce pronom relatif. Qui sans antécédent n'est donc observable, dans des syntagmes figés, que comme trace de la langue ancienne, comme dans les proverbes classiques ${ }^{3}$.

Toutefois, comme la formation des énoncés pseudo-proverbiaux le montre, il arrive qu'une certaine modification de la structure interne soit acceptée, malgré la présence d'une structure archaïque. Dans ce cas, les éléments archaïques sont en général maintenus, alors que les autres constituants deviennent des cibles de substitution lexicale. Ainsi, au lieu de qui dit Noël dit cadeaux, nous pouvons former d'autres séquences comme qui dit Noël dit sapins, ou bien même, qui dit grève dit perturbation, qui dit Bordeaux dit vin etc.

Jusqu'ici, nous avons compris que le pronom relatif qui est à garder comme tel, alors que les cases $\mathrm{X}$ et $\mathrm{Y}$ sont modifiables lexicalement, tout au moins. Alors, qu'en est-il pour les V1 et V2 ?

\subsubsection{Recherche 1 : qui + verbe}

Nous avons effectué une recherche dans 17 corpus de français, écrits et oraux, en utilisant la plateforme Orféo ${ }^{4}$. La recherche consiste en deux étapes. 
Nous avons d'abord analysé la fréquence du pronom relatif qui sans antécédent, ainsi que sa combinaison avec le verbe qui suit. Pour ce faire, nous avons sélectionné le corpus TCOF (un des corpus oraux disponibles sur Orféo). Constitué d'environ 300000 mots (soit environ 23 heures de parole), ce corpus nous permettra de connaître l'usage du pronom relatif $q u i^{5}$.

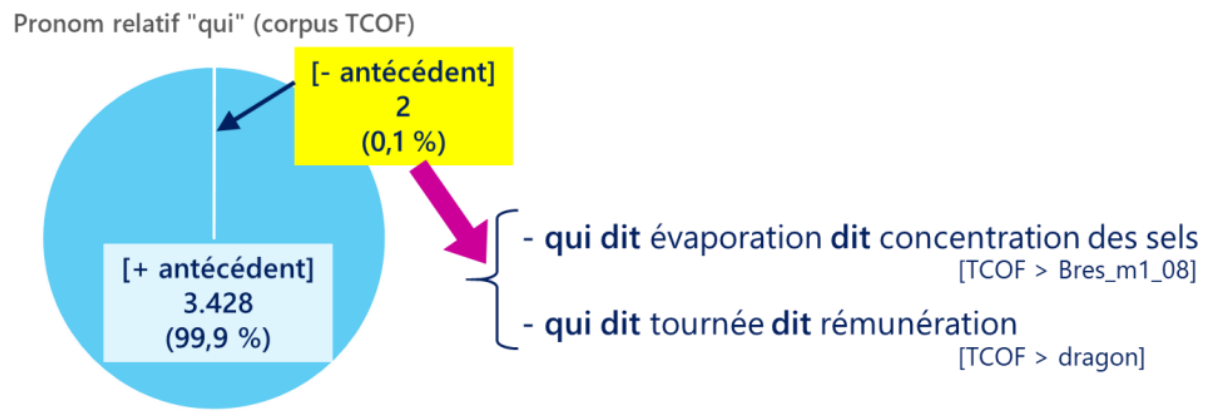

Fig. 1. Pronom relatif qui dans le corpus TCOF.

Parmi les 3430 occurrences de ce pronom relatif, il n'y en a que deux comportant qui sans antécédent. Et dans ces deux cas, le pronom relatif était suivi par dit: il s'agit de la construction qui dit $X$ dit $Y$. Ce résultat confirme l'usage très limité de ce pronom relatif sans antécédent, à l'oral tout au moins.

\subsubsection{Recherche 2 : séquences qui + dit}

Afin d'obtenir plus de résultats, nous avons choisi cette fois 17 corpus disponibles sur Orféo (dont TCOF). Nous avons obtenu 137 séquences comportant qui dit (hormis les cas du pronom interrogatif qui), dont 15 sont des cas de qui sans antécédent (dont 12 à l'oral et 3 à l'écrit). Parmi ces derniers, nous avons relevé 13 séquences en qui dit $X$ dit $Y$, dont 11 à $l^{\prime}{ }^{\prime}$ ral $^{6}$. Il est intéressant de noter que la construction semble beaucoup plus fréquente à l'oral qu'à l'écrit, malgré le côté archaïque de l'emploi de qui sans antécédent.

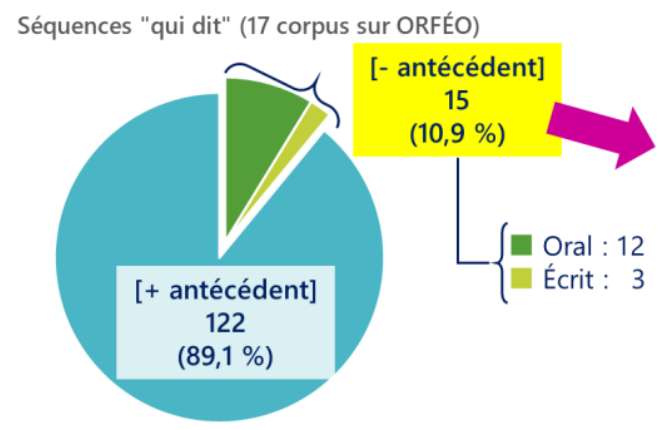

Types du V2 (17 corpus sur ORFÉO)

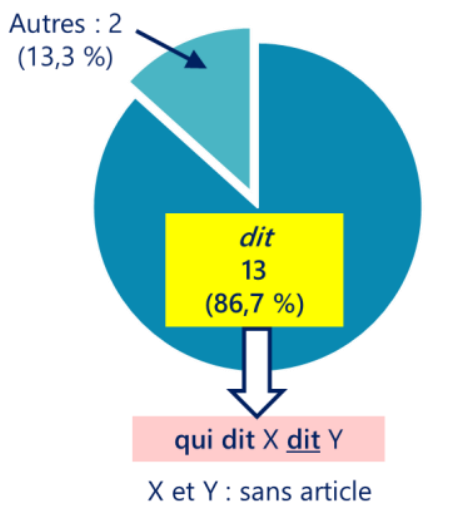

Fig. 2. Séquences qui dit dans les 17 corpus.

En ce qui concerne les cases $\mathrm{X}$ et $\mathrm{Y}$, l'article est absent pour les 13 séquences en qui dit $X$ dit $Y$. La non-actualisation des référents étant une des caractéristiques des énoncés 
(pseudo-)proverbiaux (cf. section 2.1), nous pouvons penser que les séquences qui dit $X$ dit $Y$ ont un caractère proverbial.

À partir de ces résultats, nous pouvons considérer que dans le cas des séquences commençant par qui sans antécédent : 1) le V1 doit être dit (cf. Recherche 1) et 2) le V2 aussi, (même si le V2 accepte quelques variantes: cf. Recherche 2). De plus, il est important de noter l'absence d'article qui semble généralisée pour cette construction. On en déduit donc que : 3) $\mathrm{X}$ et $\mathrm{Y}$ doivent être un syntagme nominal, mais non précédé par un article.

\subsubsection{Modèle de composition}

Ces trois conditions nous amènent à penser à la nécessité d'un modèle spécifique pour former la construction qui dit $X$ dit $Y$, plutôt que le modèle plus général : qui V1 V2. Pour la modélisation, nous adoptons l'approche constructionniste, notamment celle de Booij (2010), qui propose un schéma binaire qui représente le couplage forme-sens ${ }^{7}$. Le modèle pour qui dit $X$ dit $Y$ pourrait être établi comme ci-dessous :

\section{(3) $\left[\text { qui dit }[\mathrm{x}]_{\mathrm{Ni}} \text { dit }[\mathrm{y}]_{\mathrm{Nj}}\right]_{\mathrm{VP}} \leftrightarrow$ [saying $\mathrm{SEM}_{\mathrm{i}}$ we think of $\left.\mathrm{SEM}_{\mathrm{j}}\right]$}

La partie gauche désigne l'aspect formel de la construction. Ici, seules les cases X et $\mathrm{Y}$ restent lexicalement non-fixes (mais leur fonction syntaxique est déjà spécifiée : celle du nom, sans article), alors que les autres constituants (i.e. pronom relatif qui, V1 et V2) sont déjà fixes à ce stade. La partie droite, quant à elle, correspond à l'information sémantique que représente cette construction. Ici également, le contenu concernant $\mathrm{X}$ et $\mathrm{Y}$ (marqué comme SEM dans la formule) reste à confirmer.

Il s'agit d'une approche «output-oriented» (Booij 2010: 4): un modèle de composition est établi à partir des formes existantes, indépendamment de la compatibilité ou non avec les règles grammaticales de la langue d'aujourd'hui. Les outputs (i.e. les formes existantes) sont enregistrés dans le lexique mental. Comme Jackendoff (2002 : 187190) le relève, ces items lexicaux sont utilisés non seulement comme source de référence, mais aussi comme ressource pour développer une formule plus abstraite (i.e. modèle de composition). Jackendoff appelle ces formules «1-rules (lexicalized rules)»(p.182) pour les distinguer des règles de la grammaire. Rien n'empêche donc qu'un modèle soit basé sur une séquence figée ou non conforme à la norme grammaticale.

L'avantage de cette approche est que nous pouvons concilier la possibilité de modifier la structure interne des séquences en question avec le problème d'incompatibilité avec les règles grammaticales. Pour qu'un processus de formation soit capable de fournir de nombreuses formes, il faut un mécanisme qui permet de modifier la structure interne. Or, les constructions comme qui dit $X$ dit $Y$ n'étant pas conformes à la grammaire d'aujourd'hui, elles ne peuvent pas avoir recours à la syntaxe. En revanche, si le cadre général de la construction est préconstruit sous une formule spéciale enregistrée dans le lexique mental, il reste à remplir les cases sous-spécifiées, ce qui est une opération lexicale. C'est en considérant les modèles de composition non pas comme des règles grammaticales mais comme des unités lexicales, que la cohabitation des éléments figés et des parties libres devient possible. En effet, il existe de nombreuses constructions formées de cette manière malgré leur incompatibilité éventuelle avec les règles grammaticales : espace N2 (e.g. espace papeterie : cf. Koga 2018), $N$ après $N$ (e.g. jour après jour : cf. Magri \& Purnelle 2012) etc.

Cependant, assurer l'organisation de la structure interne des constructions sous forme d'entité lexicale ne signifie pas pour autant un refus total des modifications syntaxiques. Dans la construction qui dit $X$ dit $Y$, en effet, il reste la possibilité de mise en négation et 
d'insertion d'adverbes pour certaines positions. Il est important de savoir dans quelles conditions ces opérations syntaxiques sont acceptables.

\section{Variation et opérations syntaxiques}

\subsection{Recherches sur internet}

Afin d'analyser le lien entre la combinaison lexicale XY et les modifications syntaxiques, nous avons collecté des exemples comportant la construction qui dit $X$ dit $Y$ à l'aide du moteur de recherche Google.

La recherche a été effectuée en décembre 2019 (dernière consultation des données : le 29 décembre 2019). Nous avons tenu compte des résultats disponibles sans limiter l'analyse à une période précise, et en incluant également les résultats similaires initialement cachés par le moteur de recherche. Les textes totalement identiques apparus à plusieurs reprises (e.g. retweet) ont été supprimés manuellement. Nous avons ensuite calculé la fréquence pour les éléments remplissant la case $\mathrm{Y}$, les adverbes insérés etc.

Afin que notre recherche des données soit plus efficace et surtout faisable au niveau de la taille, nous avons sélectionné préalablement les éléments remplissant la case X. Il est souhaitable que ces éléments soient une entité qui permette aux locuteurs d'évoquer une ou des images relativement claire et certaine. Les noms propres (toponymes, noms de marque etc.) conviennent bien comme objet d'étude, mais il est nécessaire d'étudier aussi le cas des noms commun. Pour pouvoir comparer les uns et les autres, nous avons choisi trois structures nominales (deux noms propres et un nom commun), de la même catégorie : un événement qui arrive souvent (d'une manière soit périodique, soit inattendue), à savoir Journée(s) du Patrimoine, Noël et grève.

\subsection{Structure interne et possibilité de modification}

La figure suivante résume les résultats généraux pour les trois constructions choisies.

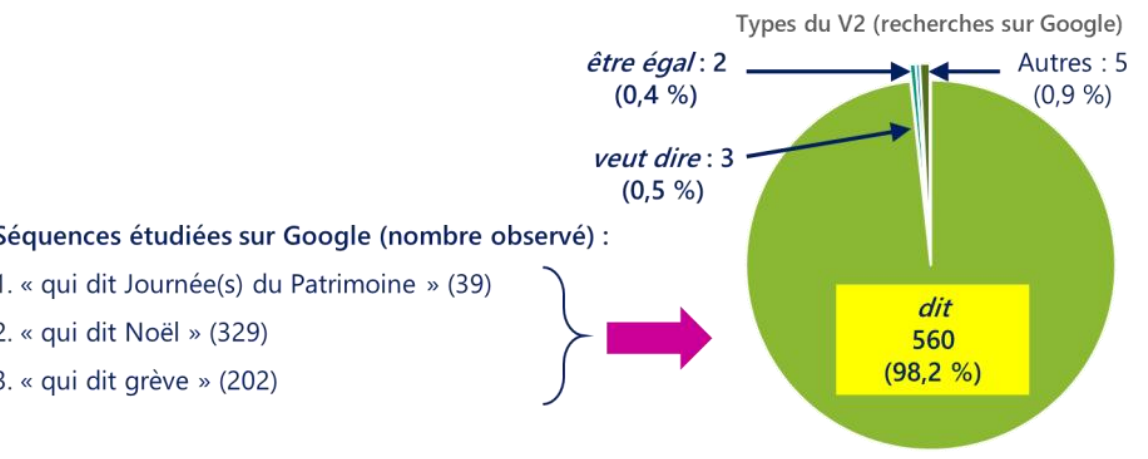

Fig. 3. Les résultats généraux pour qui dit \{Journée(s) de Patrimoine / Noël / grève\}.

Parmi les 570 séquences observées, dans la quasi-totalité des cas, le V2 était le verbe dire $(98,2 \%)$. Pour les $1,8 \%$ restant, nous pouvons constater une similitude sémantique : tous les verbes observés dans cette position concernent une évocation ou, autrement dit, un enchaînement conceptuel. À noter que les trois verbes classés dans la rubrique «autres » (i.e. signifier, évoquer, penser à) sont suivis pour chacun d'un syntagme nominal avec un article défini ou indéfini. 
En ce qui concerne les opérations syntaxiques, nous pouvons constater l'insertion d'adverbes comme dans l'exemple suivant.

\section{(4) Mais qui dit Noël, dit bien évidemment cadeaux ! ${ }^{8}$}

Il est important de noter que ces insertions ne sont possibles que pour le V2. En effet, il n'y a eu aucun cas où le V1 a été modifié par une opération syntaxique. Cela implique que la partie qui dit est complètement figée, alors que le V2 ne l'est pas.

De plus, pour le V2 même, d'autres types d'opérations ne semblent pas plausibles. En effet, nous n'avons pas pu trouver de cas où le V2 est accompagné d'un auxiliaire (?qui dit $X$ doit dire $Y)$, sauf veut dire $(0,5 \%$ des cas, voir Fig. 3). Par ailleurs, nous pouvons observer l'insertion d'un adverbe pour le V2 dans 23,3\% des cas. Il est possible que celleci soit presque la seule opération syntaxique possible pour la construction qui dit $X$ dit $Y$.

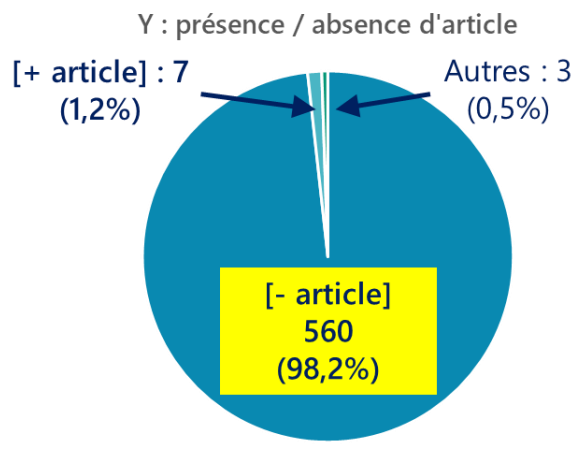

Fig. 4. La présence / absence d'article sur Y.

Quant à Y, nous pouvons constater l'absence d'article à un taux remarquable (98,2 \%). Cela correspond aux résultats pour les 17 corpus (cf. section 2.2.3). Cette tendance semble maintenue même si l'élément en position Y présente une structure complexe :

(5) Qui dit grève, dit annulation de la représentation publique du Cadre Noir, prévue ce jeudi matin entre 10 h30 à 11 h30 dans le Grand Manège. ${ }^{9}$

Il est possible que la case Y comporte plusieurs entités mises en liste (avec ou sans conjonction comme et, ou etc.), Cela concerne $15,4 \%$ des cas :

\section{Y : nombre d'entités mises en liste}

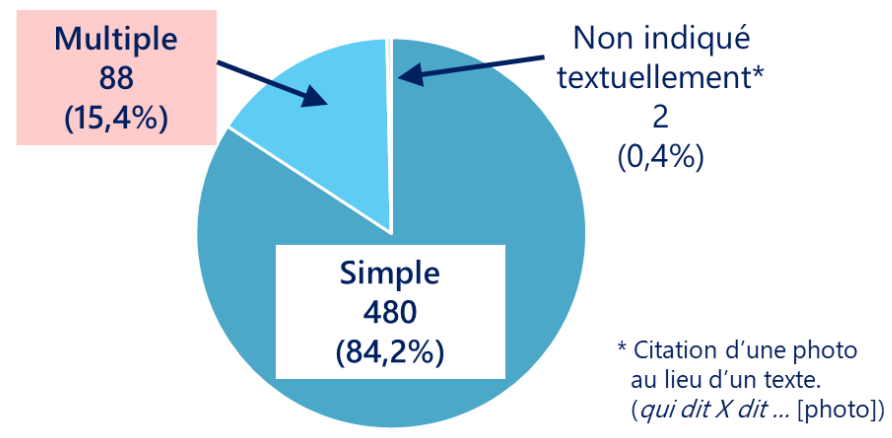

Fig. 5. Le nombre d'entités listées sur Y. 
Parmi les 88 séquences concernées, l'article est toujours absent, même si la liste est remarquablement longue comme ci-dessous :

(6) De fait, qui dit Noël dit sapin, guirlandes, déco, liste au papa noël, cadeaux, besoin d'idées cadeaux, galère d'idées cadeaux, rush dans les magasins sans idées cadeaux, achat compulsif de cadeaux n'importe quoi, fermage fort des yeux au moment de composer le code de sa carte bleue, relevé de compte plein de lignes avec, en fin de page, un nombre avec pas assez de chiffres, banquier pas content, joie de Noël en voie de disparition, engloutissage compulsif de chocolat pour faire passer la note, déprime post-engloutissage devant la silhouette irréprochable de la belle-sœur, sourire forcé de la belle-sœur devant son cadeau n'importe quoi, recherche intempestive de magie de Noël perdue, compensation à la Clairette de Die, trou noir. ${ }^{10}$

À noter qu'aucun des 19 éléments mis en liste sur la position Y n'est précédé par un article. Il existe bien un effet énumératif réalisé en général par l'enchaînement de plusieurs noms sans article, mais il faut aussi surtout considérer le fait que le modèle de composition pour qui dit $X$ dit $Y$ exige l'absence d'article.

Par ailleurs, certains exemples contiennent dit pour chaque élément ainsi listé (i.e. qui dit $X$ dit $Y 1$, dit $Y 2 \ldots$ et dit $Y n)$ :

(7) Qui dit Noël, dit joie, dit famille, dit cadeaux... Mais aussi de nombreux déchets !11

Dans cette séquence, les trois éléments énumérés sur la position Y sont tous précédés par dit, alors que ce n'est pas le cas pour le quatrième élément (i.e. de nombreux déchets) ajouté après une rupture : en revanche, cet élément mis à distance est précédé par un article indéfini ${ }^{12}$. En effet, nous pouvons constater une tendance similaire pour d'autres séquences avec une rupture, comme ci-dessous :

(8) Et qui dit Noël avec des enfants, dit aussi, ou plutôt, forcément, le Père Noël ! ${ }^{13}$

Ici également, Père Noël, posé après des éléments insérés, porte l'article défini. Toutefois, il existe aussi des contre-exemples :

(9) Qui dit Noël, dit bombance... ou mieux encore festin gourmet ! ${ }^{14}$

Dans cet énoncé, festin gourmet, ajouté après des points de suspension, est privé d'article, en accord avec l'autre élément à la même position (i.e. bombance).

Malgré toutes ces petites variations, les résultats de notre recherche confirment la supposition du modèle pour qui dit $X$ dit $Y$ proposé plus haut (cf. 3 ).

\subsection{Combinaisons lexicales et types d'adverbes}

\subsubsection{Qui dit Journée(s) du Patrimoine dit $Y$}

Maintenant, nous allons analyser les combinaisons lexicales pour $\mathrm{X}$ et $\mathrm{Y}$ ainsi que les adverbes éventuellement insérés dans les trois constructions choisies.

$\mathrm{La}$ figure suivante résume les tendances observées pour la construction qui dit Journée(s) du Patrimoine dit $Y$ : 
[Journées du Patrimoine] Y : Types de mots

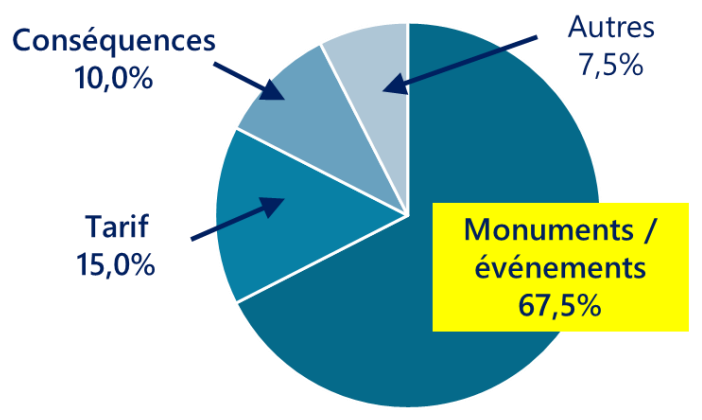

$\begin{array}{ll}\text { [+ adverbe] : 32,5 \% } & \\ \text { - ne ... pas forcément } & 5 \\ \text { - aussi } & 4 \\ \text { - forcément } & 1 \\ \text { - nécessairement } & 1 \\ \text { - obligatoirement } & 1 \\ \text { - souvent } & 1\end{array}$

(nombre observé)

Fig. 6. Les résultats pour qui dit Journée(s) du Patrimoine.

Les résultats impliquent une forte association conceptuelle entre le mot Journée(s) $d u$ Patrimoine et le lexique en rapport avec les lieux ou événements dont on parle pendant cette manifestation culturelle. Nous pouvons observer des mots comme musées, visites, vieilles choses etc. On voit aussi des mots concernant la tarification (e.g. gratuité) ainsi que des mots sur les conséquences qui se produisent (notamment files d'attente).

Quant à l'insertion d'adverbes, nous pouvons la constater dans $32,5 \%$ des cas pour le V2, parmi lesquels ne ... pas forcément est le plus nombreux :

(10) Ce week end, ce sont les fameuses "Journées du Patrimoine"! Et qui dit "Journées du Patrimoine", ne dit pas forcément longues files d'attente interminables dans les musées, sorties classiques à l'hôtel de Sully, au Château de Versailles ou au Louvre, lieux fabuleux par ailleurs. ${ }^{15}$

Comme cet exemple l'implique, la construction peut être utilisée non seulement pour indiquer une association idéelle typique, mais aussi pour dire quelque chose qui va à l'encontre d'idées préconçues. À cet égard, l'adverbe aussi, constaté également dans les résultats, peut avoir le même rôle. Au contraire, les adverbes comme forcément, nécessairement etc. reviennent à renforcer ces idées préconçues.

\subsubsection{Qui dit Noël dit Y}

Pour cette séquence, nous avons obtenu des résultats beaucoup plus nombreux et plus diversifiés par rapport au cas que nous venons de voir : 


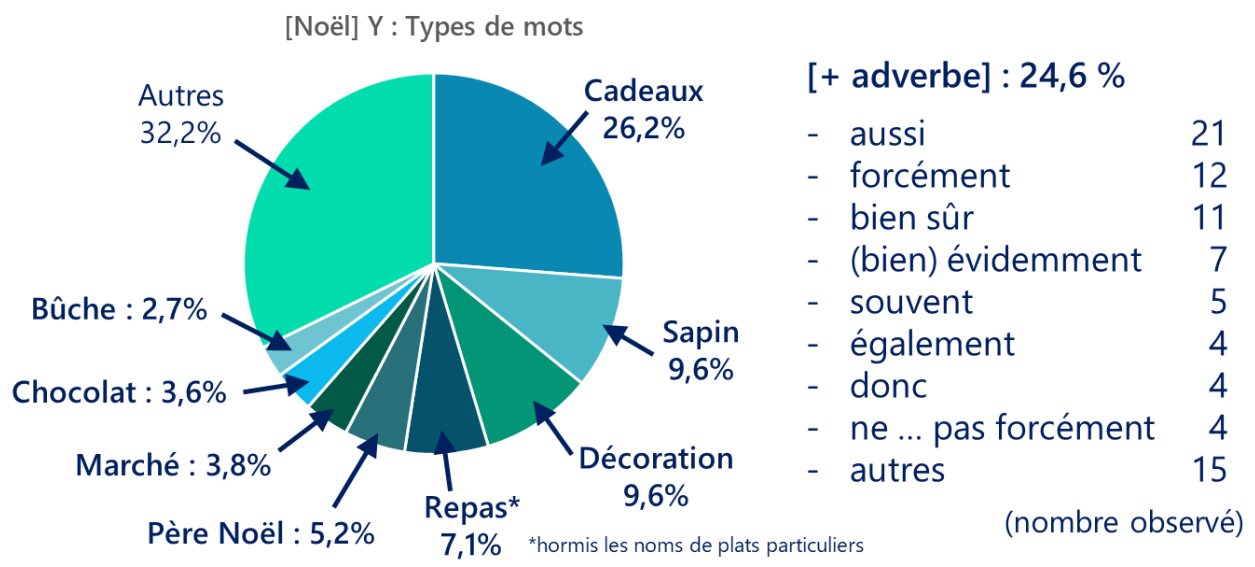

Fig. 7. Les résultats pour qui dit Noël.

La fréquence de cadeau $(x)$ et ses variantes est relativement saillante (mais loin d'être dominante) pour cette construction $(26,2 \%)$, suivie des lexiques relatifs à sapin $(9,6 \%)$, à décoration $(9,6 \%)$ à repas $(7,1 \%$, hormis les noms d'aliments ou de plats particuliers comme foie gras) etc. En ce qui concerne les adverbes insérés, la répartition est légèrement différente de celle de Journée(s) du Patrimoine: les adverbes qui renforcent les idées préconçues (i.e. forcément, bien sûr, bien évidemment etc.) sont plutôt nombreux.

Cependant, les adverbes proposant un ajout (i.e. aussi, également etc.), souvent accompagnés de la conjonction mais, sont toujours fréquents. Dans ce cas, l'image typique de Noël est affirmée avec condition. Autrement dit, l'objectif du locuteur n'est en fait pas d'affirmer ou de renforcer les idées préconçues, mais plutôt de les nuancer :

(11)Et qui dit Noël dit cadeaux, bien sûr mais aussi... papier cadeau. ${ }^{16}$

\section{(12) Qui dit Noël, dit cadeaux mais pas n'importe quel cadeau ! ${ }^{17}$}

Dans l'exemple (11), la locution adverbiale bien sûr fonctionne comme marque de concession. L'adverbe aussi, quant à lui, sert à associer un concept moins attendu (c'est-àdire un concept qui attire moins l'attention des gens en général) à l'image de Noël. Pour le cas de (12), il s'agit de donner une condition pour affirmer l'association idéelle entre Noël et cadeaux.

\subsubsection{Qui dit grève dit Y}

La troisième séquence est légèrement moins fréquente que les cas de Noël, mais montre quelques résultats bien contrastés : 


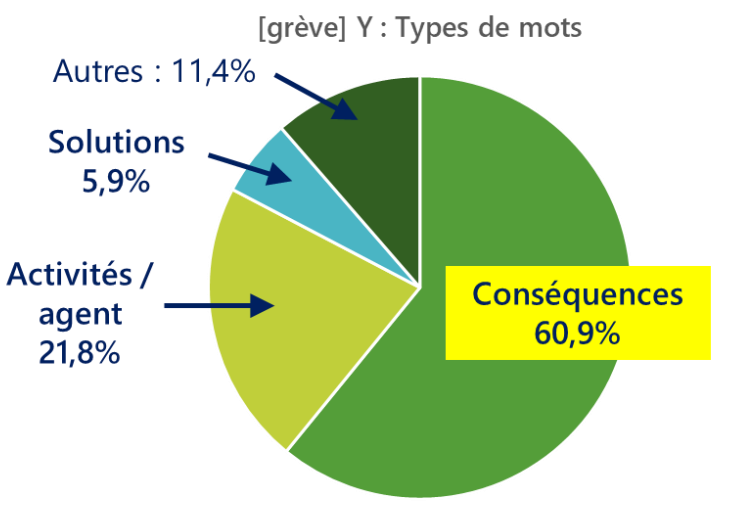

$\begin{array}{lr}\text { [+ adverbe] : 19,2 \% } & \\ \text { - donc } & 10 \\ \text { - aussi } & 8 \\ \text { - ne ... pas } & 5 \\ \text { - forcément } & 3 \\ \text { - (bien) souvent } & 3 \\ \text { - également } & 2 \\ \text { - ne ... pas forcément } & 2 \\ \text { - autres } & 6\end{array}$

(nombre observé)

Fig. 8. Les résultats pour qui dit grève.

Le lexique remplissant la case Y peut être classifié en trois catégories : les mots désignant des conséquences (souvent négatives) que cause la grève (perturbations, bouchons, galère, désagréments etc.); les mots sur les actions ou agents en rapport (manifestation, blocage, syndicat etc.) et les mots exprimant des solutions pour se débrouiller contre la situation (système $D$, covoiturage etc.). La fréquence et la variation lexicale de la première catégorie est significative dans cette construction $(60,9 \%)$. Cela implique que le mot grève évoque aux locuteurs de nombreuses conséquences et éventuellement des solutions contre elle.

Au niveau des adverbes insérés, nous pouvons observer une tendance différente de celle observée pour qui dit Noël. En effet, parmi les types d'adverbes relevés, la fréquence des adverbes de certitude (e.g. forcément) ou de fréquence (e.g. souvent) n'est comparativement pas très élevée, alors que l'adverbe donc (qui n'était pas très fréquent dans les deux premières constructions étudiées), et les adverbes d'ajout (e.g. aussi) sont comparativement un peu plus fréquents. Par ailleurs, il existe cinq négations simples (ne ...pas), qui n'étaient pas présentes dans les deux constructions précédentes.

(13) Elle déplore devoir continuer à payer son abonnement mensuel, malgré les conditions "car qui dit grève ne dit pas fin des contrôles les jours où le service est assuré, ce serait trop beau". ${ }^{18}$

Il ne s'agit pas de renforcer les idées préconçues sur la grève, ni de proposer un autre point de vue mais de ne pas associer le concept (i.e. fin des contrôles...) à l'image de la grève.

Nous venons de voir que, dans les trois constructions étudiées : 1) le lexique remplissant la case Y représente un lien idéel typique (c'est-à-dire une association idéelle largement partagée entre les membres de la communauté socio-culturelle) avec le concept marqué par le référent sur $X$, et que 2) l'insertion d'adverbes est possible pour le V2 des trois constructions, mais la fréquence d'insertion et le type d'adverbe varient selon les constructions.

Dans la section suivante, nous nous intéresserons à la fonction discursive de la construction qui dit $X$ dit $Y$ et au rôle des adverbes éventuellement insérés.

\subsection{Indicateur d'idées conventionnelles}

La construction qui dit $X$ dit $Y$ sert à exprimer un lien conceptuel entre les deux (ou plus) entités. Ce lien représente des images «typiques » (i.e. largement partagées) d'un concept. 
Nous pouvons supposer dans la construction qui dit $X$ dit $Y$ un rôle d'indicateur d'idées conventionnelles d'une communauté des locuteurs.

L'enchaînement idéel représenté par la construction qui dit $X$ dit $Y$ se rapproche de la notion de «stéréotypes » proposée dans des travaux antérieurs (cf. Fradin \& Marandin 1979, Fradin 1984, Lehmann \& Martin-Berthet [1998] 2008, Anscombre 2001 etc.). Par stéréotype, ils entendent l'ensemble des propriétés les plus attendues d'un concept. Le stéréotype «fonctionne comme le préconstruit dans un discours » (Fradin \& Marandin 1979 : 82). Partagé entre les membres d'une communauté socio-culturelle, le stéréotype est donc une « idée conventionnelle » (Lehmann \& Martin-Berthet [1998] 2008: 60). Par conséquent, cela peut varier d'une communauté socio-culturelle à l'autre.

Le cas de qui dit Noël dit Y montre bien cet aspect conventionnel de l'enchaînement idéel, car l'image typique de Noël dépend largement des communautés auxquelles les locuteurs appartiennent. Notre résultat indique que plusieurs concepts peuvent être associés à cet événement comme éléments composant d'idées conventionnelles. En rassemblant les éléments observés sur la case Y (i.e. cadeaux, sapin, décoration, repas, Père Noël etc.), nous pourrions établir une image typique de Noël. Par ailleurs, s'il s'agissait d'une communauté étrangère (surtout non chrétienne), les idées conventionnelles ainsi représentés seraient différents ${ }^{19}$.

Les adverbes éventuellement insérés sur le V2 expriment l'attitude des locuteurs vis-àvis des idées conventionnelles. Ici, nous pouvons constater trois types de réaction :

Le premier type concerne l'affirmation ou le renforcement des idées préconçues. Les adverbes de certitude comme forcément, bien sûr, bien évidemment servent à le faire. Parmi les trois constructions étudiées, ce type d'adverbe est relativement fréquent dans qui dit Noël dit $Y^{20}$. Il est possible que les idées conventionnelles concernant le concept Noël tendent à être reçues comme telles (c'est-à-dire sans objection) par les locuteurs.

Le deuxième type de réaction est de nuancer. Cela peut être réalisé avec négation : ne ... pas, ne ... pas forcément etc. Par exemple, l'énoncé suivant signale qu'il y a des cas opposés aux idées conventionnelles des Journées du Patrimoine (i.e. gratuité) :

(14) Mais attention qui dit Journée du Patrimoine ne dit pas forcément gratuité sur tous les sites. ${ }^{21}$

En mettant le V2 en négation, il devient possible de traiter les idées moins facilement associées au concept proposé. C'est la syntaxe qui permet à la construction d'exprimer non seulement les cas qui correspondent aux idées conventionnelles, mais aussi ceux qui s'y opposent.

Le troisième type est la proposition d'ajout. Celle-ci est réalisée par des adverbes comme aussi, également etc. Comme nous l'avons déjà relevé (cf. section 3.3.1), l'effet réalisé par ces adverbes peut être similaire à celui du deuxième type, dans la mesure où les deux proposent un autre point de vue que les associations idéelles typiques proposées :

(15)Comme un goût de déjà-vu. Écoles fermées, trains annulés, pas de crèche, transports perturbés... C'est le même refrain. Hier, plusieurs usagers ont de nouveau dî prendre leur mal en patience pour affronter la deuxième journée d'appel à la grève. Et qui dit grève, dit aussi système $D .^{22}$

Comme système $D$ semble un effet secondaire de la grève, le lien idéel entre les deux concepts est moins attendu, par rapport aux concepts liés plus directement comme écoles fermées, trains annulés etc., indiqués au préalable (quoique ces éléments soient présents en dehors de la construction qui dit $X$ dit $Y$ ). Les adverbes d'ajout permettent de faire remarquer la présence d'autres concepts qui concernent aussi des idées conventionnelles de la grève. 
Dans tous les cas, il s'est révélé que la construction qui dit $X$ dit $Y$ joue un rôle d'indicateur d'idées conventionnelles (ou de contre-exemples d'idées conventionnelles). Une fois établie cette chaîne conceptuelle, rien n'empêche de la développer davantage. En effet, dans les données que nous avons collectées, il existe un certain nombre de séquences enchaînées, comme l'exemple suivant :

(16) Et qui dit réforme, dit mécontentement et qui dit mécontentement en France, dit grève et qui dit grève dit syndicats (ou l'inverse). ${ }^{23}$

Ici, les trois constructions qui dit $X$ dit $Y$ s'enchaînent pour construire en trois étapes une association basée sur des idées conventionnelles.

Le développement d'une chaîne idéelle peut se faire par un autre moyen. La séquence citée dans (6) est un bon exemple. Là, il n'y a qu'une seule construction qui dit $X$ dit $Y$, mais les éléments réalisés par Y s'entassent d'une manière quasi interminable.

\section{En guise de conclusion}

Nous avons étudié les caractéristiques de qui dit $X$ dit $Y$, à partir de données collectées dans des corpus et sur internet. En permettant la cohabitation des parties figées et des parties sous-spécifiées, le modèle de composition propose un cadre préconstruit pour former des énoncés pseudo-proverbiaux. Dans cette construction, l'emploi archaïque du pronom relatif qui est préservé comme partie figée. Les résultats de notre recherche dans les corpus et sur internet confirment que le prénom relatif sans antécédent est accompagné dans la quasitotalité des cas de l'élément verbal dit (V1). Quant au deuxième dit (V2), la possibilité de substitution reste limitée à certains verbes synonymes de dire.

La construction accepte l'insertion d'un adverbe pour le V2, mais il est difficile de réaliser les autres types d'opérations syntaxiques. Pour le V1, la modification des verbes est presque impossible. Compte tenu de ces caractéristiques, nous pourrions considérer que le modèle de composition pour former cette construction présente un caractère plutôt lexical.

La construction qui dit $X$ dit $Y$ n'est pas simplement un énoncé pseudo-proverbial mais un indicateur d'idées conventionnelles. Nous avons constaté trois types de réaction vis-àvis de ces images préconstruites: l'affirmation (ou le renforcement), la proposition de contre-exemples et l'ajout. Cela peut être réalisé par l'intermédiaire des adverbes.

Par ailleurs, cette construction a une fonction discursive similaire à celle des énoncés du type La France, c'est le vin. Il serait nécessaire de savoir si les trois types de réaction que nous venons de relever sont également observables dans ce type d'énoncés, et, s'il existe d'autres constructions qui jouent un rôle d'indicateur d'idées conventionnelles, comme qui dit $X$ dit $Y$.

\section{Références bibliographiques}

Anscombre, J.-C. (2001) Le rôle du lexique dans la théorie des stéréotypes, Langages, 142, pp.57-76. ----- (2003) Les proverbes sont-ils des expressions figées ?, Cahiers de lexicologie, 82, pp. 159-173.

Booij, G. (2010) Construction Morphology, Oxford : Oxford University Press.

Conenna, M. (1988) Sur un lexique-grammaire comparé de proverbes, Langages, 90, pp.99-116.

Fradin, B. (1984) Anaphorisation et stéréotypes nominaux, Lingua, 34, pp.325-369.

Fradin, B. \& Marandin, J.-M. (1979) Autour de la définition : de la lexicographie à la sémantique, Langue française, 43, pp.60-83.

Gross, G. (1996) Les expressions figées en français, Paris : Ophrys. 
Jackendoff, R. (2002) Foundations of Language, Oxford / New York : Oxford University Press.

Koga, K. (2018) Un espace papeterie n'est-il pas une (simple) papeterie ? : composition binominale sous-catégorisante, Actes du Congrès Mondial de Linguistique Française - CMLF 2018, 16p. DOI : https://doi.org/10.1051/shsconf/20184608004, publié le 9 juillet 2018.

Lehmann, A. \& Martin-Berthet, F. (1998) Introduction à la lexicologie : sémantique et morphologie, 3ème edition (2008), Paris : Armand Colin.

Magri, V. \& Purnelle, G. (2012) Mot à mot, brin par brin : les suites [Nom préposition Nom] comme motifs, JADT 2012 - Actes des $11^{\text {es }}$ Journées internationales d'Analyse statistique des Données Textuelles, pp.659-673.

Mejri, S. (2001) La structuration sémantique des énoncés proverbiaux, L'information grammaticale, 88 , pp.10-15.

----- (2005) Figement absolu ou relatif : la notion de degré de figement, Linx, 53, pp.183-196.

Schapira, Ch. (2000) Proverbe, proverbialisation et déproverbialisation, Langages, 139, pp.81-97.

Tamba, I. (2000) Formules et dire proverbial, Langages, 139, pp.110-118.

\footnotetext{
${ }^{1}$ Nous pouvons constater ici « certains effets particuliers destinés à attirer l'attention du lecteur » (Gross 1996 : 20). Il semble que la déformation des énoncés figés (i.e. défigement) d'une manière inattendue fasse partie d'une « activité ludique » (ibid.).

2 « http://madame.lefigaro.fr/beaute/1-kg-24-h-chrono-010611-162070», le $1^{\text {er }}$ juin 2011.

${ }^{3}$ Conenna (1988) rapporte que, à la différence de qui en français, le pronom relatif chi en italien peut être employé sans antécédent.

${ }^{4}$ La plateforme établie dans le cadre du projet ANR 12-CORP-0005 (dirigé par Jeanne-Marie

Debaisieux). Recherche effectuée le 4 mai 2019. Voici les 17 corpus disponibles sur la plateforme :

- Corpus écrits (6) : ANNODIS, Chambers-Rostand, CoReMe, Est Républicain, Frantext (hors théâtre et poésie) et Scientext 2010.

- Corpus oraux (11) : CFPP2000, CLAPI, CRFP, C-ORAL-ROM, FLEURON, French Oral Narrative, HUSIANYCIA, OFROM, TCOF, TUFS et VALIBEL.

Pour plus de détails, voir le site du projet : «www.projet-orfeo.fr »

${ }^{5}$ De plus, les discours spontanés qui constituent ce corpus nous permettront d'éviter des cas d'élaboration stylistique qui sont potentiellement présents à l'écrit. Ce point est d'autant plus important que la construction étudiée ici concerne des énoncés proverbiaux, souvent liés aux connaissances encyclopédiques.

${ }^{6}$ Parmi les 11 cas observés à l'oral, il y a un cas où la seconde partie de la séquence apparaît après une rupture, recommencé non pas par dit $Y$, mais par ça veut dire $Y$. Compte tenu de la similitude sémantique du verbe, nous le traitons pour le moment comme une variante de dit $Y$. Voir aussi la section 3 pour la variation de V2.

${ }^{7}$ Strictement parlant, le schéma est en trois parties, avec la section «phonologie » en plus, mais ici, nous adoptons la version binaire simplifiée pour rendre l'explication plus compréhensible.

${ }^{8}$ « https://naona-shop.com/comment-trouver-le-meilleur-cadeau-de-noel-pour-femme/ », le 18 octobre 2019. Nous avons mis nous-même certaines parties en gras. Idem pour les autres exemples cités infra.

9 «www.saumur-kiosque.com/infos_article.php?id_actu=31203», le 2 juin 2016.

10 « https://boycottcitoyen.org/2019/12/12/boycott-a-la-surconsommation-de-noel-episode-2-unsapin-na-rien-a-faire-dans-un-salon/ », le 12 décembre 2019.

${ }_{11}^{11}$ «www.estrepublicain.fr/edition-luneville/2019/12/02/luneville-apprendre-a-faire-son-proprecalendrier-grace-a-recyclune », le 9 décembre 2019.

${ }^{12}$ Il est possible, cependant, que l'article indéfini de soit inséparable de nombreuse. Si cela est le cas, il faudra considérer de nombreux (de nombreuses) comme une séquence figée, à distinguer des cas d'un syntagme nominal avec un article indéfini (cf. 8).

${ }^{13}$ «www.tendanceradis.com/single-post/2016/12/19/noel-zerodechet », le 19 décembre 2016.

${ }^{14}$ «www.soyuz.digital/2012/12/01/top-5-pages-daccueil-noel/», le ${ }^{\text {er }}$ décembre 2012.

15 « http://culturesurlezinc.over-blog.com/article-22908146.html», le 18 septembre 2008.
} 
16 « https://reporterre.net/Un-Noel-emballant-avec-Pakekado-maitre-en-furoshiki », le 14 décembre 2019 .

${ }^{17}$ « https://vivonslenergieautrement.fr/special-noel-5-cadeaux-intelligents-qui-font-du-bien-a-laplanete/ », le 19 décembre 2019.

18 « www.francetvinfo.fr/economie/transports/sncf/greve-a-la-sncf/greve-a-la-sncf-vous-avez-partagevos-astuces-pour-faire-face-a-1-absence-de-trains_2687770.html », le 4 avril 2018.

${ }^{19}$ En effet, pour les Japonais par exemple, l'image du chocolat est moins attendue pour Noël, car ils n'ont pas de tradition d'en consommer à cette occasion.

${ }^{20}$ En même temps, le nombre de cas où l'adverbe n'est pas inséré sur le V2 est aussi élevé pour cette construction (83 \%). Dans ce cas, il n'y a pas d'effet de renforcement idéel, mais au moins une affirmation des stéréotypes proposés.

21 «www.marieclaire.fr/maison/25e-journees-europeennes-du-patrimoine,200317,1140448.asp », le $1^{\mathrm{er}}$ septembre 2008 .

22 « www.ladepeche.fr/2019/12/11/grand-sud-comment-les-usagers-sadaptent-au-quotidien-avec-lagreve,8596675.php », le 11 décembre 2019.

23 « http://videgrenierecrit.canalblog.com/archives/2019/12/25/37891333.html », le 25 décembre 2019. 